\title{
Creación en narrativa, otra cara de la investigación literaria
}

\author{
Aleyda Gutiérrez Mavesoy ${ }^{1}$ \\ Universidad Central de Bogotá
}

"La creación es la más eficaz de todas las escuelas de paciencia y lucidez. Es también el testimonio trastornador de la única dignidad del hombre: la rebelión tenaz contra su condición, la perseverancia en un esfuerzo considerado estéril".

Albert Camus

"Me gusta considerar a todo escritor como un creador que se propone, imitando a Dios, la creación de un mundo nuevo".

Emile Zola

\section{Resumen}

Este artículo gira en torno a la relación creación e investigación. La hipótesis central es que ambos desarrollan un mismo proceso, al menos un proceso similar, y sólo se diferencian en el resultado final. Para comprobar esto, parte de la delimitación del concepto de creación literaria. Luego, confronta la idea supuesta de oposición entre la creación y la investigación, para finalmente concluir que la relación entre ambos es el proceso.

Palabras clave: creación narrativa, investigación literaria, escritura, lenguaje.

\section{Abstract}

This paper is about the relationship between the creative writing and the investigative studies. The central idea is both of them have the same process and they just are different on the final result. To prove that, it starts with the notion of creative writing evolution. Then, it argue about the apparently opposition between the narrative knowledge and the scientific knowledge, to finally conclude the relationship between them is the process.

Key words: narrative creation, literary research, writing, language.

\footnotetext{
${ }^{1}$ Maestría en Literatura Hispanoamericana del Instituto Caro y Cuervo. Profesora de la Universidad Central de Bogotá, en el área de Humanidades. Ha trabajado mayormente sobre el lenguaje con énfasis en el desarrollo de los procesos de lectura y escritura en el ámbito universitario. Paralelamente ha venido estudiando el campo de la literatura en distintas áreas de su ejercicio académico. E-mail: aleydag34@ yahoo.com.mx
} 
La creación literaria es una de las artes que más se ha resistido a su sistematización. En el mundo académico universitario colombiano es común encontrar carreras de música, artes plásticas, cine, danza o teatro, pero no de creación literaria. Si bien es cierto que muchos escritores han mencionado el trabajo, la disciplina, el laborioso cuidado del escritor con el lenguaje, los años de escritura, corrección, re-escritura -otras veces, la aceptación de la pérdida de material escrito, porque no pasa la prueba de la revisión-, continuamos pensando en la escritura de obras literarias como algo ajeno a la Academia: se puede formar para ser músicos, cineastas, bailarines, actores, artistas plásticos, pero no escritores. Aún la palabra "creación" o "creador" se pronuncia con reserva. Esto debido, entre otras, por la idea de que lo que gobierna a la escritura de obras literarias es el "impulso" o la "intuición"; es decir, todavía predomina la concepción idealista de la escritura: entendida desde ahí como un acto irracional en el que el artista es dominado por su arte; no es producto de su elaboración racional. No obstante, la creación literaria tiene mucho de pulsión, pero también mucho de ejercicio consciente con el lenguaje. En el proceso de escritura hay un problema por resolver, una intención estética que necesita una forma, un deber con la palabra que lleva a la indagación constante: hay en la creación mucho de investigación. La división radical entre creación e investigación basada en la dicotomía racionalidad-irracionalidad es parcial, además de forzosa. Para ahondar en este planteamiento se hace necesario que consideremos la cuestión paso a paso. En esta explicación, partamos de la delimitación conceptual del término "creación narrativa", para, después, profundizar en la relación creación-investigación en literatura.

El lenguaje es la herramienta fundamental de la que se vale el hombre para organizar la experiencia; al mismo tiempo, dispone de este recurso para construir otras realidades posibles. De entre las distintas modalidades del lenguaje, el relato se configura como la forma de organización de la experiencia por excelencia. La narración ordena los acontecimientos y acciones de los sujetos-personajes para configurar el mundo que propone. No obstante, la narración de ficción realiza una construcción simbólica del universo real que le permite la bifurcación de los mundos en múltiples posibilidades de sentido, que, en su materialización, genera un modelo figurado de las intenciones de los actores, las causas de lo que ocurre y los procesos internos de la acción humana desde una construcción específicamente estética. De este modo, lo que distingue la creación narrativa de la narrativa en general es que está orientada siempre por una preocupación central: la cualidad artística o literaria de las configuraciones que del mundo elabora. 
Ahora bien, el término "creación narrativa" obedece a una necesidad conceptual básica de delimitación: mientras en el mundo anglosajón suele ser corriente la aplicación de la expresión creative writing ("escritura creativa"), para referirse a la escritura de obras literarias, desde la corriente europea se prefiere la denominación "creación narrativa".

En el mundo hispano predomina el uso europeo, debido, por un lado, a la tradición latina, y por el otro, a que el término "escritura creativa" puede conducir a ambivalencias. El ámbito académico nuestro, en primer lugar, hace referencia al trabajo pedagógico de los maestros para que los estudiantes incrementen sus competencias en el dominio de la lengua. En segundo lugar, se refiere a las formas en que se produce el discurso con predominio de la función estética, bien sea desde el discurso de la publicidad, el periodismo u otras disciplinas afines. En tercer lugar, el empleo que de la discursividad narrativa hacen las ciencias, para explicar fenómenos sociales o naturales. Finalmente, desde la psicología, se ha distinguido entre el conocimiento producto del pensamiento lógico matemático y el conocimiento que deviene del pensamiento narrativo. Por todo ello, utilizar el término "escritura creativa" genera un problema conceptual evidente: la ambivalencia conceptual del vocablo.

En nuestro medio es reconocida, y corresponde al uso común del mundo académico, la expresión "creación narrativa" para delimitar, aclarar y especificar el tipo de discurso narrativo con fines primordialmente estéticos. Pero, ¿de dónde viene el término "creación narrativa"? Los griegos emplearon la expresión "fabricar" para referirse a la producción poética, y la de "descubridor", para el artista. Ello debido a que los conceptos de creador o creación implicaban "libertad de acción", rasgo que debía permanecer ajeno a las artes, puesto que, bajo su concepción, un artista no crea sino que se rige por las leyes y normas que buscan imitar la naturaleza. No obstante, ello no tiene una valoración negativa. Para los griegos, la Naturaleza era perfecta por lo que la acción de los hombres debía estar encaminada a parecerse a ella, descubrir las leyes y someterse a ellas; luego, el artista era un descubridor, no un inventor. Por algo, "canon" quiere decir "medida". Sólo el poeta salía de este estándar, porque era el que "fabricaba", ofrecía cosas nuevas al traer un nuevo mundo a la vida, ya que no estaba sujeto a ninguna serie de leyes. Para Platón, la poesía imita una cosa de tal manera que crea aquello que imita, es decir, hace que una cosa llegue a existir de nuevo.

Si bien los romanos utilizaban los vocablos creatio ("creación") y creare ("crear"), su uso se restringía al referente creator, sinónimo 
de padre y creator urbis, fundador de una ciudad. Por lo demás, se mantenía la concepción griega del artista como "imitador". Durante la época medieval los pensadores religiosos llegaron más allá: tomando el sentido romano de la palabra creator llegaron a designar "creación" al acto que Dios realiza a partir de la nada: creatio ex nihilo. Del mismo modo, al tomar el vocablo griego "fabricar" y adjudicarle el sentido de la época, igualan la poesía a las otras artes. El poeta podía fabricar, pero no crear, ya que, como las otras artes, seguía unas normas y unas leyes en su proceso de elaboración (rima, ritmo, etc.).

Durante el Renacimiento los artistas dieron diversos nombres a su proceso de producción de la obra de arte. El artista "inventa", "preordena", "emplea formas que no existen en la naturaleza", "plasma su visión", "configura un mundo nuevo", "inventa lo que no es", "hace surgir de la nada", "produce una transformación"... Las distintas denominaciones tratan de eludir el término "creación", por la implicaciones teológicas - para entonces, todavía recientes- que hay en él. Bien entrada la Ilustración se acepta que el poeta inventa, construye según su estilo, y sobre todo, crea algo nuevo, "tal como lo hace Dios".

Aunque la idea de que la creación era un atributo exclusivo del artista surgió en el siglo XVII, es con los románticos, en pleno siglo XIX, que se afianza y se convierte en un rasgo definitorio del arte. Se hizo común la presunción de que la creación era una particularidad del artista, y creador llegó a ser sinónimo del mismo. En el siglo XX la expresión se aplicó a todas las formas simbólicas de la cultura: se habló de creación en las ciencias, la política, la vida cotidiana. El término se relacionaba con la fabricación de cosas nuevas - no a partir de la nada-, y es de aquí que surge la distinción anglosajona. El significado derivado del latín creatio hace referencia al proceso, para distinguirlo del atributo -capacidad que tienen todos los seres humanos para crear-, por lo cual se introduce el término creative ("creativo"). Por ello, hoy en día, en psicología y pedagogía es corriente el uso de referentes como creativo o creatividad para hacer distinción de la producción de la obra artística.

Vale la pena decir que con lo que respecta a la creación narrativa nos enfrentamos a un problema, más o menos similar. Gerard Genette (1988) reflexiona en torno al problema de los géneros. Según él, la nominación es producto de una interpretación posterior y no una propuesta de los griegos. En primer lugar, en el libro tercero de la República, Platón sólo considera las formas (lexis) de la poesía "narrativa". Ateniéndose a su definición "todo poema es relato (diégesis) de sucesos pasados, presentes o por venir", excluye la poesía lírica, 
puesto que bajo su concepción no hay más poema que el representativo (mimético). Dentro de la diégesis, estima tres formas posibles: puramente narrativa -relato puro- (ditirambo), mimética -presentar los personajes en acción- (tragedia o comedia) y mixta (epopeya). En segundo lugar, lo que Platón llama lexis, Aristóteles designa como "manera de imitar". Según Genette, la expresión más exacta sería la de "modo", ya que "forma", en el sentido tradicional, daría cuenta sólo de un aspecto.

Por otra parte, Genette presenta el tránsito que se da entre la clasificación de Platón y la de Aristóteles de la siguiente manera:

\begin{tabular}{|c|c|c|}
\hline Objeto $\quad$ Modo & Dramático & Narrativo \\
\hline Superior & Tragedia & Epopeya \\
\hline Inferior & Comedia & Parodia \\
\hline
\end{tabular}

De acuerdo con este esquema, ni Platón ni Aristóteles consideraron la cuestión de los géneros, ni tampoco incluyeron la poesía lírica dentro de su clasificación de los modos de la poesía. Esta vez es en el periodo medieval, con Diomedes, que se renombran como géneros (genera) los tres modos platónicos. A partir de allí se empiezan los intentos por introducir la poesía no representativa en dicha clasificación. Se propone como salida el hecho de que así como la narrativa imita acciones, la lírica imita emociones; por lo tanto, la poesía lírica, al imitar sentimientos reales, genera ficción, y por tanto, es también imitación. Sobre la base de este argumento se produjo la clasificación que hoy día conocemos: lírica, narrativa, dramática. El cuadro correspondiente sería:

\begin{tabular}{|c|c|c|c|}
\hline Platón & Narrativo & Mixto & Dramático \\
\hline Aristóteles & & Narrativo & Dramático \\
\hline Antigüedad & & Épico & Dramático \\
\hline Modernidad & Lírico & Narrativo & Dramático \\
\hline
\end{tabular}

Sin embargo, después de mediados del siglo XX en adelante, no sólo la explicación de la lírica se transforma, sino también el concepto de narrativa. Hoy en día se concibe la narrativa como fenómeno comunicacional que contempla tanto el acto de narrar como el producto, entendido como producción de significaciones y resignificaciones del mundo. De este modo, las narraciones permiten estructurar y organizar la inteligibilidad del mundo, tanto en la vida cotidiana como en los 
espacios del saber tradicionalmente académico y no sólo artístico. Es bien sabido que la narración se utiliza como recurso epistemológico de las ciencias sociales y de otras instancias, puesto que, en un sentido amplio, las teorías se constituyen como narraciones, que son formas estructuradoras del conocimiento, por tanto, productoras de sentido.

Sumado a ello, la narrativa es entendida como práctica simbólica que adquiere sentido en un contexto social, pero que a la vez apoya la disposición de dicho contexto social como espacio de significación que involucra a los sujetos. Esto es, la narrativa en su dimensión comunicativa tiene una naturaleza indexical y reflexiva (es un fenómeno de comunicación y a la vez una orientación epistemológica particular). En conclusión a este apartado, puede inferirse que las diferentes prácticas de la narración (en el folklore, la etnografía, etc.), dependen de las formas y procedimientos regidos por la intención que orienta su elaboración. Lo que hoy en día se conoce como narrativa abarca diversos usos en espacios de la vida cotidiana, académica y artística. Es por ello que el campo de la "creación narrativa" requiere, efectivamente, de una delimitación conceptual tangible. Si bien aceptamos que de la narrativa pueden darse diversos usos, debemos admitir que en la literatura prima una intención estética -sin que haya menoscabo de una intención ética- en la producción de la obra narrativa.

De acuerdo con estas delimitaciones previas, necesarias, podemos afirmar que el campo de estudio de la creación narrativa es "la producción de una existencia de ficción". Es decir, desde la creación narrativa se orienta la composición global a una finalidad estética. En la producción de un universo narrativo se privilegia la forma como se da vida a unos seres de ficción, cuyos destinos materializan las evaluaciones críticas que de la condición humana realiza el creador en el mundo ficcional. Unido a lo anterior, la creación narrativa atraviesa tres momentos: imita, descubre, inventa el mundo: imita la realidad al conservar los límites de lo verosímil; descubre las leyes y la belleza que lo configura como realidad, e inventa lo que no existía, la nueva realidad. Por ello mismo, el campo de estudio se estructura en torno al acto de narrar, sus productos, sus formas y procedimientos, además del universo que configura. También, haciendo paráfrasis de los griegos, podemos decir que al imitar-descubrir-inventar está realizando, de otro modo, la investigación que describe, explica y prescribe la naturaleza.

Ahora bien, el estudio del lenguaje como ciencia empieza a ser considerado a partir de la propuesta teórica de Ferdinand de Saussure. El paradigma del estructuralismo encuentra su versión en literatura a partir de los formalistas rusos, quienes establecen que el objeto de estudio es la literariedad. "Lo que nos caracteriza [...] es el deseo de 
crear una ciencia literaria autónoma a partir de las cualidades intrínsecas de los materiales literarios. [...] el objeto de la ciencia literaria no es la literatura, sino la literariedad, es decir, lo que hace de una obra dada una obra literaria" (Universidad de Antioquia, 2004). La concentración de los formalistas en el cómo del arte, es decir, en la forma en que los escritores producen la obra de arte ha sido el mayor aporte de esta corriente a la consolidación en el ámbito académico de los estudios literarios.

Es posible afirmar, a su vez, que la búsqueda del cientificismo en los estudios literarios es una presunción particular del siglo XX. La teoría, la crítica y la historia literaria han estado siempre en la historia de Occidente; desde los griegos hasta nuestros días la preocupación por el fenómeno literario ha encontrado diversas explicaciones desde las distintas disciplinas: filosofía, psicología, sociología y en las últimas década, los estudios culturales. Estos últimos han pretendido definir, describir, clasificar y entender el hecho literario como un hecho social. Sin embargo, la búsqueda de la validez científica de los planteamientos teóricos, críticos o históricos ha tenido en el siglo XX su mayor asidero, ya que es en este siglo cuando más paradigmas en torno al lenguaje, y su inclusión en distintos campos del saber, se han presentado. Para el caso, valga un ejemplo:

En los últimos diez años se ha producido un importante cambio en la manera de abordar el tema de las identidades sociales. Obviamente ligado a lo que se dio en llamar "el giro lingüístico" en las ciencias sociales, pero precisando mucho más la relación entre identidades y discursos, este cambio hace hincapié no sólo en el origen discursivo de las identidades (algo ya avanzado por el interaccionalismo simbólico americano y por Althusser y Foucault a principios de la década de los 70s, y muy bien sintetizado por Laclau y Mouffe en los 80s), sino también en su origen narrativo. (Vila, 1996).

De acuerdo con los distintos paradigmas del siglo XX, se ha establecido como tarea de esta ciencia el estudio de los medios de construcción de las obras literarias, su método, la descripción, clasificación y explicación de los fenómenos literarios bajo un marco teórico que lo sustente. Los formalistas rusos, Mijail Bajtín, el estructuralismo, el post-estructuralismo, la sociocrítica, la estética de la recepción, la hermenéutica, los estudios de género, los estudios culturales, los estudios postcoloniales, todos estos paradigmas, han abordado la literatura con un enfoque científico, aunque han tomado prestado de otras ciencias los métodos para el estudio de la obra literaria: 


\begin{abstract}
Nosotros no teníamos, ni tenemos ninguna doctrina o sistema acabado. En nuestro trabajo científico, apreciamos la teoría como hipótesis de trabajo con cuya ayuda se indican y comprenden los hechos: se descubre el carácter sistemático de los mismos gracias al cual llega a ser material de estudio. No nos ocupamos de las definiciones que nuestros epígonos buscan ávidamente y tampoco construimos las teorías generales que los eclécticos encuentran tan agradables. Establecemos principios concretos $y$, en la medida en que pueden ser aplicados a una materia, nos atenemos a ellos (Eichembaum, citado por Todorov, 1991: s. p.)
\end{abstract}

Entonces, en el establecimiento de los estudios literarios como ciencia hay un afán por validar los procesos singulares que se llevan a cabo en el campo de la literatura, tan diferentes al campo de las ciencias naturales. Esta intención por validar la investigación literaria de la teoría, la crítica y la historia ha redundado en cierto menoscabo de la investigación literaria del creador. Al intentar alcanzar la dimensión de objetividad, al buscar separarse de su objeto, niega implícitamente ese estatuto a la labor del creador. La tarea del teórico, del crítico, del historiador o del creador, puede llegar a coincidir en el proceso, en el método o hasta en el mismo objeto, lo que les diferencia es el producto de su investigación. Se ha preferido eludir esta cuestión, porque ello significa borrar los límites entre uno y otro.

Veamos cómo es esto posible. En principio, la creación literaria tiene el mismo énfasis hacia la rigurosidad, profundidad, precisión y objetividad, ya que la pesquisa literaria del escritor, o la del investigador, apunta hacia un mismo objeto, no el real, la literatura, sino el objeto de estudio: la obra que deviene en obra literaria. Es bien sabido que en nuestro tiempo, aún antes, los recursos, técnicas y estrategias utilizadas por cualquier investigador científico suelen ser asimiladas por muchos escritores para recopilar información para su análisis y génesis en el proceso de creación.

Por supuesto, los escritores -y muchos críticos así lo promueven- se han quedado entre las musas, la inspiración o la genialidad, pocas veces aceptan el carácter "labrador" de la escritura, el trabajo consciente, reiterativo, cuando no constante del "tallador de palabras". Ya se utiliza como un cliché el enfrentamiento del escritor a la crítica: dos mundos incompatibles. A pesar de ello, podemos reiterar que hay en la creación mucho de talento, pero también mucho de disciplina, de diálogo con los otros y con la herencia literaria que los lleva a la renovación constante. Por tanto, requiere de una fuerte investigación en el campo y no sólo de la pulsión interna. 
Si aceptamos esto, podemos pasar ahora a la cuestión de los saberes que produce cada uno. Para ello, retomemos a Lyotard (1992). Según este filósofo, el saber científico exige el predominio de un juego específico de lenguaje, el denotativo. Ese rasgo de objetividad sólo puede encontrarse en él, ya que el criterio de aceptabilidad de un enunciado es su valor de verdad. Para que se produzca un enunciado de verdad, la interrogación y la prescripción forman parte de la argumentación dialéctica que llevan a un enunciado a su instancia denotativa. Por el contrario, el saber narrativo no valora la cuestión de su propia legitimación, no le interesa producir enunciados de verdad, sino de verosimilitud, hecho que le lleva a su acreditación por la pragmática de su transmisión. Sin embargo, esta distinción ya no es tan clara. Las obras, los planteamientos de los mismos escritores de la segunda mitad del siglo XX en adelante, han generado una crisis del estatuto de verdad y de las distancias entre los saberes. Los escritores desde distintas disciplinas y artes han quebrado los límites entre la creación-la crítica-la teoría.

Veamos cómo ocurre esto dentro del ámbito literario. Dos son los acontecimientos fundamentales en este espacio: la hibridez de los campos del saber y el énfasis en la metaficción. La hibridez de las esferas del saber es bastante común hoy en día; entre el periodismo, la historia, la literatura, la psicología y la pedagogía hay muchos vasos comunicantes. Verbigracia, después de la década del 50 en el siglo pasado se desarrolló una preocupación fuerte por la historia en América Latina, hasta el punto de provocar el surgimiento de una tendencia de la novela que en nuestro continente se denominó Nueva Novela Histórica: no le bastó con ser verosímil, buscó ser veraz, más aún que la historia oficial:

Pero ¿qué sucede cuando un autor no puede escapar a la historia? ¿Cuándo no puede olvidar, a voluntad, lo aprendido? [...] 0 en otras palabras: ¿qué sucede -qué hacer- cuando no se quiere eludir la historia y sin embargo al mismo tiempo se desea alcanzar la poesía? Quizás la solución sea no plantearse una alternativa, como Borges, y no eludir la historia, como Usigli, sino tratar de conciliar todo lo verdadero que pueda tener la historia con lo exacto que pueda tener la invención. (Del Paso, 1988: 641)

Las incursiones del periodismo en la literatura son bastantes a su vez; el testimonio es hijo directo de la hibridez entre el periodismo y la literatura. La novela testimonial hace camino en nuestro continente, producto de esa búsqueda iniciada por el periodismo entre la denuncia crítica de la realidad y la propuesta estética de la literatura 
para la composición artística de dicha investigación. Ernest Hemingway, Elena Poniatowska, Tomás Eloy Martínez, entre algunos de los más reconocidos, son un buen ejemplo de la hibridez entre el periodismo y la literatura.

No es necesario hablar de las conexiones entre las diversas artes, entre las más nombradas la relación cine-literatura. En la psicología ha cobrado especial importancia el recurso de la narrativa como estrategia terapéutica, mientras que en pedagogía se han descubierto nuevas formas de conocer a través de ella. Sin lugar a dudas, el lenguaje en su expresión poética ha salido de la esfera de la literatura y habita en otros campos, insospechados en el pasado. Hay que hacer notar que este fenómeno no acontece sólo en el ámbito literario. En el espacio científico también se ha producido una fractura de sus límites. Es posible identificar, al menos, dos acontecimientos importantes. En primer lugar, el establecimiento del carácter discursivo del lenguaje científico. En segundo lugar, y directamente relacionado, la relatividad del principio de verdad.

Los filósofos de la postmodernidad han comprobado que el discurso científico es uno más de entre los diversos discursos posibles en el mundo cultural. Asimismo, han comprobado que hay en él una lógica inmanente a toda forma del lenguaje y que no le es de suyo exclusivo. Hay en todos ellos una fuerte reacción a la omnipresencia del positivismo que dominó a las ciencias por mucho tiempo. Por ejemplo, Juan José Sebreli menciona, en el Prólogo a la edición del 2003 de su libro Buenos Aires, vida cotidiana y alienación, que la crítica se ha centrado en el tipo de fuentes que utiliza para sus planteamientos, periódicos, revistas y novelas. Afirma que ha seleccionado este tipo de fuentes intencionalmente, porque es su forma de contrarrestar el positivismo reinante y asfixiante de las ciencias sociales, especialmente en la sociología.

En segundo lugar, los avances en las reflexiones en torno a las ciencias. Se ha podido comprobar que la narración es usada en las ciencias sociales como objeto de estudio, pero también como método de reporte de los resultados de la investigación y como sistema explicativo de los mismos. Así, la narración es el objeto, es el método y es la epistemología. El observador de cualquier realidad, por más objetivo que sea, se para desde un paradigma, una mirada que rige la mirada; luego, toda observación es parcial, y en cierta medida, subjetiva. Los teóricos han hecho estallar el concepto de La Verdad y en su lugar han instaurado las verdades, parciales, restringidas y paradigmáticas, como quienes las enuncian. 
Como corolario, podemos afirmar ahora que en las condiciones actuales del ámbito académico en el que confluyen todas las formas de la cultura y del saber para imbricarse, resquebrajar los límites, establecer nuevas formas y hermanar los opuestos, la creación y la investigación literaria son dos caras de un mismo proceso. Lo que los distingue no es el camino, es el punto de partida y de llegada. Tal como lo presentía Delacroix hace ya un buen tiempo, y que se aplica tanto para el escritor como para el investigador:

Existe un acuerdo en que lo que se ha dado en llamar la creatividad de los grandes artistas no es sino el modo de ver, ordenar e imitar la naturaleza peculiar de cada uno de ellos.

\section{Bibliografía}

Contursi, María Eugenia \& Ferro, Fabiola. (2000). La narración, usos y teorías. Buenos Aires: Norma.

Del paso, Fernando. (1988). Noticias del Imperio. Bogotá: La Oveja Negra.

Genette, Gerard. (1988). “Géneros, "tipos”, modos”. En Garrido Gallardo, Miguel. (comp.), Teoría de los géneros. Madrid: Arco/libros.

Lyotard, Jean Francois. (1992). La condición postmoderna: informe sobre el saber. Barcelona: Planeta Agostini. Trad. Mariano Antolín Rato.

Perus, Francoise. (1976). Literatura y sociedad en América Latina: el modernismo. La Habana: Casa de las Américas.

Rubiano, Roberto. (2006). Alquimia de escritor. Bogotá: Icono.

Sebreli, Juan José. (2003). Buenos Aires, vida cotidiana y alienación; Buenos Aires, ciudad en crisis. Buenos Aires: Sudamericana.

Tatarkiewics, Wladyslaw. (1988). Historia de seis ideas: arte, belleza, forma, creatividad, mímesis, experiencia estética. Madrid: Tecnos.

Todorov, Tzvetan. (1991). Teoría de la literatura de los formalistas rusos. México: Siglo Veintiuno editores. 1a ed. 1970.

Universidad de Antioquia. (2004). "La historiografía en los estudios literarios". En Departamento de Lingüística y Literatura, Facultad de Comunicaciones, Curso de Historiografía Literaria. Recuperado entre el 25 de abril y el 2 de mayo de 2007. Disponible en http:// comunicaciones.udea.edu.co/historiografia/ciclo2/introduccion/historiografi a_i.pdf

Valles Calatrava, José. (1994). Introducción histórica a las teorías de la narrativa, Almería:Universidad de Almería.

Vila, Pablo. (1996). “Identidades narrativas y música. Una primera propuesta para entender sus relaciones", Revista Transcultural de Música. Recuperado entre el 5 de abril y el 2 de mayo de 2007. Disponible en http://www.sibetrans.com/trans/trans2/vila.htm 
